\title{
BMJ Open Hymenoptera venom allergy: work disability and occupational impact of venom immunotherapy
}

\author{
Giulia Paolocci, ${ }^{1}$ Ilenia Folletti, ${ }^{1}$ Kjell Torén, ${ }^{1,2}$ Giacomo Muzi, ${ }^{1}$ Nicola Murgia ${ }^{1,2}$
}

To cite: Paolocci G, Folletti I, Torén $\mathrm{K}$, et al. Hymenoptera venom allergy: work disability and occupational impact of venom immunotherapy. BMJ Open 2014;4:e005593. doi:10.1136/bmjopen-2014005593

\section{- Prepublication history for this paper is available online. To view these files please visit the journal online (http://dx.doi.org/10.1136/ bmjopen-2014-005593).}

Received 30 April 2014 Revised 21 July 2014 Accepted 22 July 2014

\begin{abstract}
${ }^{1}$ Section of Occupational Medicine, Respiratory Diseases and Toxicology, University of Perugia, Perugia, Italy

${ }^{2}$ Department of Occupational and Environmental Medicine, Sahlgrenska University Hospital, Göteborg, Sweden
\end{abstract}

Correspondence to Dr Giulia Paolocci; giulia.paolocci@unipg.it

\section{ABSTRACT}

Objectives: Little is known about the Hymenoptera venom allergy impact on work ability and the effect of venom immunotherapy (VIT) on work. The objective of this study was to evaluate the prevalence and predictors of work disability in patients treated with VIT and the effects of VIT on occupational functioning. Methods: 181 patients, aged 18-71 years, treated with VIT while working, were investigated by questionnaire. Participants were classified into employed and self-employed and, based on work exposure to Hymenoptera, into three risk categories: high risk, occasionally high risk and low risk. Work disability was defined as having to have changed jobs/ tasks and/or suffered economic loss because of Hymenoptera venom allergy. Predictors of work disability were assessed in logistic regression models. Results: 31 (17\%) patients reported work disability. Being self-employed and having the severe reaction at work were associated with work disability $(p<0.01)$. Having a high-risk job for exposure to Hymenoptera was a significant predictor of work disability (OR 2.66, $95 \% \mathrm{Cl} 1.04$ to 6.75 ). $24 \%$ of patients referred a positive effect of VIT on work. Determinants of the positive effect of VIT on work were having a high-risk job for exposure to hymenoptera (OR $3.60,95 \% \mathrm{Cl}$ 1.52 to 8.51 ) and having already concluded VIT (OR 2.82, $95 \% \mathrm{Cl} 1.30$ to 6.14 ).

Conclusions: Hymenoptera venom allergy could determine work disability. Patients with Hymenoptera venom allergy having a high-risk job for exposure to Hymenoptera seem to have higher risk of work disability and refer more frequently a positive effect of VIT on work.

\section{INTRODUCTION}

Hymenoptera venom allergy affects approximately $5 \%$ of the general population and can provoke severe systemic or lifethreatening reactions. ${ }^{1} \quad$ Epidemiological studies indicate a prevalence of self-reported systemic anaphylactic sting reactions between $0.3 \%$ and $7.5 \%,{ }^{2}$ and mortality due to insect sting ranging from 0.03 to 0.48 fatalities per 1000000 population per year. ${ }^{3}$

\section{Strengths and limitations of this study}

- In the current study, for the first time, work disability and occupational effects of Hymenoptera venom allergy were studied in a group of patients in working age.

- The results suggest that Hymenoptera venom allergy has an impact on work, causing work disability.

- Self-employed workers and workers at high risk of sting seem to be at higher risk of work disability related to Hymenoptera venom allergy.

- The small number of participants could be considered a shortcoming of this survey.

- Individuals not undergoing venom immunotherapy are not considered in this study.

Since the late 1970s, venom immunotherapy (VIT) has provided allergic participants with protection from fatal anaphylaxis and prevented about $90 \%$ of all reactions to stings. ${ }^{4}$ Patient compliance for long-term continuation of VIT often decreases, making VIT an effective but challenging therapy. ${ }^{5}$ Besides, even with VIT, for most patients as well as for their families, an anaphylactic reaction after a Hymenoptera sting is a very traumatic event, and the fear of a subsequent life-threatening episode may affect the emotional, social and occupational behaviour of the affected individual. ${ }^{6}$ Recently, a diseasespecific questionnaire, the Vespid Allergy Quality of Life Questionnaire, was designed and validated for assessing health-related quality of life in patients with anaphylactic responses following yellow jacket stings. The survey showed that patients experienced quality-of-life impairment especially because of the emotional distress associated with having to be constantly on the alert while leading their everyday 'normal' lives. ${ }^{7}$

One important part of the everyday 'normal' life is work. Any factor that would affect occupational functioning could lead to work disability. Many definitions of work disability have been applied over the past 
decades. Recently, changing jobs or tasks, or having suffered loss in working days or in finance because of illness have often been used to define work disability in participants with respiratory diseases. ${ }^{8}{ }^{9}$ Prevalence, incidence, determinants and VIT effects of allergic sting reactions have been largely investigated in selected occupational groups such as gardeners and beekeepers. ${ }^{6}$ However, little is known about Hymenoptera venom allergy impact on work ability.

The primary aim of this study is to evaluate the prevalence and predictors of work disability in a group of patient treated with VIT. The secondary outcome was to assess the effects of VIT on occupational functioning.

\section{METHODS}

Population and questionnaire

The clinical charts of 364 patients treated with VIT from 1997 to 2011 at the Perugia University Hospital, Italy, were reviewed. In these participants VIT was prescribed because they reported a history of systemic severe reaction and a documented sensitisation to the respective insect with either skin tests and/or specific serum IgE tests. Prior to starting VIT, all the patients enrolled in this study underwent the diagnostic protocol according to European Academy of Allergy and Clinical Immunology guidelines. ${ }^{7}$ Serum IgE for Hymenoptera venom were assessed by Phadia 100 (Phadia AB, Uppsala, Sweden); skin tests were performed with venom extract of Apis mellifera Vespula sp., Polistes sp. Vespa crabro from Stallergenes (Antony, France).

Selection of venom to be used in immunotherapy was based on the identification of the species of Hymenoptera involved and on cross-reactivity between venoms, where the exact identification of the responsible insect was not possible. The extracts used for VIT were from Stallergenes (Antony, France), Alk-Abello (Hørsholm, Denmark) and Anallergo (Firenze, Italy). One hundred and twenty-seven participants were treated with aqueous extract, 54 with depot.

For all the participants a slow protocol of desensitisation was used. The time required to reach the generally adequate maintenance venom dose of $100 \mu \mathrm{g}$ was 10 15 weeks and immunotherapy was recommended for at least 5 years. ${ }^{7}$

Of the 364 patients undergoing VIT, 183 were excluded from the study because they were retired, housewives or students. A total of 181 patients, aged 18-71 years, and treated with VIT while working, were eligible for the study. A questionnaire administered by a physician was used to collect data on demographic characteristics (age, gender, address), the offending insect and severity of symptoms after Hymenoptera stings, graded according to the Mueller classification. Circumstances regarding the Hymenoptera sting leading to the anaphylactic reaction and time from severe reaction were also reported. Participants were classified as employed or self-employed and, according to a classification based on work exposure to Hymenoptera used in a previous study, ${ }^{1}$ into three categories: high risk (individuals usually working outdoors or where Hymenoptera live: farmers, gardeners, fire fighters, truck drivers, masons, beekeepers, garbage collectors); occasionally at high risk (sometimes working outdoors, eg, plumbers); and at low risk (working indoors, eg, clerks).

Work disability was defined as an affirmative response to at least one of these key questions:

1. Did you change work because of Hymenoptera sting reaction?

2. Did you change your job tasks because of Hymenoptera sting reaction?

3. Did you suffer economic loss because of Hymenoptera sting reaction?

The definition of work disability was formulated on the basis of previous surveys about work disability, widely available in the literature. ${ }^{8}{ }^{9}$ To assess the effect of VIT, specific questions were asked about a possible positive, negative or indifferent effect of the treatment on work. As other questionnaires were not available on this topic, a panel of allergologists and occupational physicians reviewed and proposed the questions used in this study about possible indicators of a treatment-related positive effect, such as not having changed jobs or tasks and feeling safer at work, or indicators of a negative effect, such as economic loss, changes of job or task and changes in working time. Participants with negative or indifferent effect of VIT on work were classified as not having a positive effect on work.

The study protocol was approved by the Ethical Committee of Umbria Region (CEAS). All the patients gave their informed and written consent to participate in this study.

\section{Statistical analysis}

Differences between patients with and without work disability were evaluated by $\chi^{2}$ test or Fisher's exact test, as appropriate, for categorical variables, and using Mann-Whitney $\mathrm{U}$ test or $\mathrm{t}$ test for continuous variables. Predictors of work disability and positive or negative VIT effects on work were assessed in logistic regression models, adjusted for gender and age, choosing as independent variables those showing a different distribution across the groups in the univariate analysis. The goodness of fit of the logistic regression models was confirmed by the Hosmer-Lemeshow test. ${ }^{10}$ All the analyses were performed using SPSS statistical software, V.20.0 (SPSS, IBM Corporation, New York, USA).

\section{RESULTS}

All 181 patients treated with VIT while working answered the questionnaire. Among them, $68 \%$ were still undergoing VIT and $32 \%$ had concluded the therapy. The mean age was 49 years, $19 \%$ were female and more than half of the patients lived in the countryside. Thirty-one patients $(17 \%)$ reported work disability. No patient 
reported a complete work change because of Hymenoptera venom allergy, $10(5 \%)$ reported having had to change job tasks, and 25 (14\%) reported economic loss because of Hymenoptera venom allergy, 4 (2\%) reported both conditions. The characteristics of the study population, classified according to work disability due to Hymenoptera venom allergy, are shown in table 1. No differences were found in distribution of gender, residence, mean age, stinging insect and severity of the allergic reaction between the two groups. In participants with work disability, Vespula was responsible for $45 \%$ of the reactions, and $45 \%$ experienced a systemic allergic reaction grade 4, according to Mueller's classification.

Fourteen patients (45\%) with work disability and 26 (17\%) patients without work disability experienced a sting reaction to Hymenoptera during shift $(p<0.01)$. Patients with work disability were mostly workers at high risk of work exposure to Hymenoptera while participants without work disability were mostly at low risk $(68 \%$ vs $41 \%, \mathrm{p}<0.001$; table 1 ).

Forty-four participants (24\%) reported a positive effect of VIT on work, 93 had no impact (51\%) and 44 were negative $(24 \%)$. The characteristics of the population, classified by the presence or absence of the positive impact of VIT on work, are displayed in table 2. Participants reporting a positive effect of VIT on work were more frequently at higher risk of work exposure to Hymenoptera, self-employed workers and have experienced the allergic reaction at work (table 2). Evaluating the same characteristics presented in table 2, there were no significant differences between those with a negative impact of VIT and the other participants (those with a positive or indifferent impact on work).

In the logistic regression analysis, adjusted for gender and age, significant predictor of work disability was having a high-risk job for exposure to Hymenoptera (OR 2.66, 95\% CI 1.04 to 6.75 ; table 3 ).

Table 1 Characteristics of the patients with Hymenoptera venom allergy treated with VIT, with or without work disability

\begin{tabular}{|c|c|c|c|}
\hline & $\begin{array}{l}\text { Participants with } \\
\text { work disability } \\
\mathrm{n}=31\end{array}$ & $\begin{array}{l}\text { Participants without } \\
\text { work disability } \\
n=150\end{array}$ & p Value \\
\hline Female, n (\%) & $6(19.4)$ & $37(24.7)$ & NS \\
\hline Age, mean $\pm S D$ & $49.4 \pm 9.9$ & $47.1 \pm 10.9$ & NS \\
\hline Living in the countryside, $\mathrm{n}(\%)$ & $20(64.5)$ & $98(65.3)$ & NS \\
\hline \multicolumn{4}{|l|}{ VIT, n (\%) } \\
\hline Ongoing & $21(67.7)$ & $101(67.3)$ & \multirow[t]{2}{*}{ NS } \\
\hline Concluded & $10(32.2)$ & $49(67.3)$ & \\
\hline \multicolumn{4}{|l|}{ Treated with VIT for, n (\%) } \\
\hline Apis mellifera & $11(35.5)$ & $48(32)$ & NS \\
\hline Vespula sp. & $14(45.2)$ & $52(34.7)$ & NS \\
\hline Polistes sp. & $3(9.7)$ & $38(25.3)$ & NS \\
\hline Vespa crabro & $3(9.7)$ & $12(8)$ & NS \\
\hline \multicolumn{4}{|l|}{ Type of VIT extract } \\
\hline Aqueous & $23(74.2)$ & $104(69.3)$ & \multirow[t]{2}{*}{ NS } \\
\hline Depot & $8(25.8)$ & $46(30.7)$ & \\
\hline \multicolumn{4}{|l|}{ Mueller reaction grade, $\mathrm{n}(\%)$} \\
\hline I & $6(19.4)$ & $27(18)$ & \multirow[t]{4}{*}{ NS } \\
\hline II & $4(12.9)$ & $30(20)$ & \\
\hline III & 7 (22.6) & $30(20)$ & \\
\hline IV & $14(45.2)$ & $63(42)$ & \\
\hline Ever stung before the reaction, $\mathrm{n}(\%)$ & 26 (83.9) & $110(73.3)$ & NS \\
\hline Other severe Hymenoptera reactions, n (\%) & $1(3.2)$ & $12(8)$ & NS \\
\hline Years after the first severe reaction, mean $\pm S D$ & $9 \pm 6.7$ & $7.1 \pm 5.7$ & NS \\
\hline \multicolumn{4}{|l|}{ Having the severe reaction, $\mathrm{n}(\%)$} \\
\hline At work & $14(45.2)$ & $26(17.3)$ & $<0.01$ \\
\hline During sport & $1(3.2)$ & $4(2.7)$ & NS \\
\hline During hobby activity & $9(29)$ & $52(34.7)$ & NS \\
\hline At home & $6(19.4)$ & $44(29.3)$ & NS \\
\hline In car/motorcycle & $3(9.7)$ & $24(16)$ & NS \\
\hline \multicolumn{4}{|c|}{ Work-related risk of exposure to Hymenoptera, n (\%) } \\
\hline High & $21(67.7)$ & $53(35.3)$ & \multirow[t]{3}{*}{$<0.01$} \\
\hline Occasionally high & $5(16.1)$ & $35(23.3)$ & \\
\hline Low & $5(16.1)$ & $62(41.3)$ & \\
\hline Self-employed worker, n (\%) & $6(51.6)$ & $42(28)$ & $<0.01$ \\
\hline
\end{tabular}


Table 2 Characteristics of the patients with regard to the impact of VIT on work

\begin{tabular}{|c|c|c|c|}
\hline & \multicolumn{2}{|c|}{ Impact of VIT on work } & \multirow[b]{2}{*}{ p Value } \\
\hline & $\begin{array}{l}\text { Positive } \\
n=44\end{array}$ & $\begin{array}{l}\text { Negative or indifferent } \\
n=137\end{array}$ & \\
\hline Female, n (\%) & $12(27.3)$ & $31(22.6)$ & NS \\
\hline Age, mean $\pm S D$ & $49.7 \pm 11.7$ & $46.8 \pm 10.3$ & NS \\
\hline \multicolumn{4}{|l|}{ VIT, n (\%) } \\
\hline Ongoing & $22(50)$ & $100(73)$ & \multirow[t]{2}{*}{$<0.01$} \\
\hline Concluded & $22(50)$ & $37(27)$ & \\
\hline \multicolumn{4}{|l|}{ Treated with VIT for, $\mathrm{n}(\%)$} \\
\hline Apis mellifera & $11(25)$ & $48(35)$ & NS \\
\hline Vespula sp. & $17(38.6)$ & $49(35.8)$ & NS \\
\hline Polistes sp. & $13(22.7)$ & $31(22.6)$ & NS \\
\hline Vespa crabro & $6(13.6)$ & $9(6.6)$ & NS \\
\hline \multicolumn{4}{|l|}{ Mueller reaction grade, $\mathrm{n}(\%)$} \\
\hline 1 & $7(15.9)$ & 26 (19) & \multirow[t]{4}{*}{ NS } \\
\hline II & $6(13.6)$ & $28(20.4)$ & \\
\hline III & $12(27.3)$ & $25(18.2)$ & \\
\hline IV & $19(43.2)$ & $58(42.3)$ & \\
\hline Ever stung before the reaction, $\mathrm{n}(\%)$ & $38(86.4)$ & $102(74.5)$ & NS \\
\hline Other severe Hymenoptera reactions, n (\%) & $2(4.5)$ & $11(8)$ & NS \\
\hline Years after the first severe reaction, mean $\pm S D$ & $7.7 \pm 5.4$ & $7.3 \pm 6.1$ & NS \\
\hline Having the severe reaction at work, n (\%) & $17(38.6)$ & $23(16.8)$ & $<0.005$ \\
\hline \multicolumn{4}{|c|}{ Work-related risk of exposure to Hymenoptera, $n(\%)$} \\
\hline High & $28(63.6)$ & $46(33.6)$ & \multirow[t]{3}{*}{$<0.01$} \\
\hline Occasionally high & $5(11.4)$ & $35(25.5)$ & \\
\hline Low & $11(25)$ & $56(40.9)$ & \\
\hline Self-employed worker, n (\%) & $20(45.5)$ & $38(27.7)$ & $<0.05$ \\
\hline
\end{tabular}

Working in a high-risk job for exposure to Hymenoptera was a determinant of the positive effect of VIT on work (OR 3.60, 95\% CI 1.522 to 8.508 ) as well having already concluded VIT (OR 2.82, 95\% CI 1.30 to 6.14; table 4).

\section{DISCUSSION}

Hymenoptera venom allergy and work disability

In this study a not negligible percentage of patients with severe Hymenoptera venom allergy requiring VIT reported work disability. Our main finding is the association between occupational characteristics (jobs at a high risk of exposure to Hymenoptera) and work disability related to Hymenoptera venom allergy.

Our results suggest that the presence of systemic Hymenoptera allergy can lead to occupational problems,

Table 3 Predictors of work disability among patients undergoing Hymenoptera venom immunotherapy (VIT), adjusted for gender and age

\begin{tabular}{llll}
\hline & OR & \multicolumn{2}{l}{$95 \% \mathbf{C l}$} \\
\hline Having the severe reaction at work & 2.306 & 0.909 & 5.852 \\
Workers at high risk of exposure to & 2.655 & 1.044 & 6.754 \\
Hymenoptera & & & \\
Self-employed workers & 2.079 & 0.883 & 4.892 \\
\hline
\end{tabular}

especially when the severe reaction took place during work. This was consistent with the data in the literature. ${ }^{6}$

In our study, the percentage of workers at high risk of sting (beekeepers, farmers, truck drivers) was slightly higher $(41 \%)$ compared with workers occasionally at risk $(22 \%)$ or those with low risk of sting $(37 \%)$. Working in these high-risk jobs for sting was a significant risk factor for work disability. These findings support the hypothesis that Hymenoptera venom allergy could be considered an occupational disease. ${ }^{1}$

Work disability and decreased work productivity usually occur in other similar diseases too, such as asthma and allergic rhinitis, ${ }^{9}{ }^{11}$ especially if ocular symptoms are present. ${ }^{12}$ In studies on respiratory work disability, the exposure to risk factors, such as irritants, is the most important predictor of work disability. ${ }^{13}$ This was also the finding in this study, where workers most

Table 4 Predictors of the effect of venom immunotherapy (VIT) on work, adjusted for gender and age

\begin{tabular}{llll}
\hline Positive VIT effect & OR & \multicolumn{2}{l}{$95 \%$ CI } \\
\hline VIT concluded & 2.822 & 1.296 & 6.144 \\
Having the severe reaction at work & 1.777 & 0.721 & 4.384 \\
Workers at high risk of exposure to & 3.599 & 1.522 & 8.508 \\
Hymenoptera & & & \\
Self-employed workers & 1.323 & 0.601 & 2.912 \\
\hline
\end{tabular}


likely to be exposed to Hymenoptera were at higher risk of work disability. In other studies, blue-collar workers have been reported to be at higher risk of occupational consequences of Hymenoptera venom allergy than white-collar workers ${ }^{6}$; similar to our study the category of workers at high risk of exposure to Hymenoptera referred more work disability (change work/task and/or economic loss) than the other two groups (occasionally at high risk, and at low risk). In this study, the risk of work disability for self-employed workers was not statistically significant as expected because some authors reported that employees were usually at higher risk of health-related job loss than self-employed workers. ${ }^{14}$ The non-significant risk increase related to 'having the reaction at work' could be explained by the obvious correlation between high-risk jobs for sting and the occurrence of reaction in a population characterised by severe allergic reaction to Hymenoptera venom.

\section{VIT impact on work}

VIT is globally accepted as the treatment of choice in venom allergy. ${ }^{15}$

Although 44 patients in the current study declared a negative effect of VIT on work, caused by changing working time and economic loss due to VIT, most of our participants declared an indifferent effect of VIT on work. Individuals at high risk of sting reported a positive VIT impact on work. This will support the accepted medical approach that occupation may influence the decision to initiate VIT, also for non-life-threatening reactions. ${ }^{5}$

Another predictor of a perceived positive effect from VIT was completion of treatment. Participants who have already completed VIT are probably more aware of the long-term beneficial effects of treatment. They are less likely to report any drawbacks of VIT, such as the time spent in therapy, which can affect work and social life. A relevant number of participants reported a negative impact of VIT on work and even if we could not find any peculiar characteristic of this group, this finding deserves attention. To prevent this side effect of VIT related to the amount of time spent in therapy, it is important to underline that we have room for improvement, developing new forms and routes of VIT (eg, sublingual VIT) ${ }^{16}$ or more convenient regimens such as rush up-dosing, which may lead to improved patient compliance. Unfortunately, some studies have concluded that the accelerated VIT protocols are associated with a significant increase in the incidence of systemic reactions compared with conventional protocols. ${ }^{17}$ Moreover, such accelerated protocols may necessitate new allocations of medical services and further timetabling in relation to employment. ${ }^{6}$ Another way to reduce the reported negative effect of VIT could be to improve information about the risks related to a new reaction to Hymenoptera venom among patients selected for VIT, who are those at higher risk of severe reactions. During this study, we had the impression that information about venom-related life-threatening reaction is not homogenous in our group of patient. Stressing the efficacy of VIT for the prevention of life-threatening reaction could be a good strategy to improve the acceptance of this treatment. VIT could be considered a workplace intervention to reduce work disability, even if, as a result of the few available studies on work intervention to prevent work disability, no convincing conclusions can be drawn about the effectiveness of these interventions. ${ }^{18}$

\section{Validity issues}

The small number of participants could be considered a shortcoming of this study; however the subject is novel, the disease is rare and in the literature previous reports on work disability with similar numbers have already been published. ${ }^{8}{ }^{19}$ As the existing questionnaire about quality of life in patients with Hymenoptera venom allergy does not take into account work disability, ${ }^{20}$ we had to design our questionnaire, defining work disability as job/task change or economic loss due to the disease (in our case, Hymenoptera venom allergy). ${ }^{8}{ }^{19}{ }^{21} \mathrm{We}$ were aware of a possible recall bias, as reported in other questionnaire-based surveys. To minimise recall bias we also considered the entity of the allergic reaction according to the Mueller grading scale, as well as the presence of other severe reactions and the time from the severe reaction that push the participant to consult an allergologist. None of these variables had a significant effect on work disability. Furthermore, we preferred to use prompted questions, which are less prone to recall bias, especially for occupational exposures. ${ }^{22}$

\section{CONCLUSIONS}

This cross-sectional study suggests that Hymenoptera venom allergy has an impact on work through causing work disability. Having a high-risk job for sting appears to be a significant risk factor for work disability as well a predictor of a perceived positive impact of VIT on work. Therefore, for the prevention and treatment of patients we have to consider also the occupational burden of Hymenoptera venom allergy.

Acknowledgements The authors thank Istituto Nazionale per l'Assicurazione Contro gli Infortuni sul Lavoro (INAIL) which supported the study.

Contributors GP and NM designed the study; GP, IF and NM were responsible for data collection. NM, KT and GM managed and analysed the data. All authors participated in the interpretation and final drafting of the manuscript.

Funding This study was funded by the Section of Occupational Medicine, Respiratory Diseases and Toxicology of the University of Perugia and by Istituto Nazionale per l'Assicurazione Contro gli Infortuni sul Lavoro (INAIL).

Competing interests None.

Patient consent Obtained.

Ethics approval Ethical Committee of Umbria Region (CEAS Umbria), Italy.

Provenance and peer review Not commissioned; externally peer reviewed.

Data sharing statement No additional data are available. 
Open Access This is an Open Access article distributed in accordance with the Creative Commons Attribution Non Commercial (CC BY-NC 4.0) license, which permits others to distribute, remix, adapt, build upon this work noncommercially, and license their derivative works on different terms, provided the original work is properly cited and the use is non-commercial. See: http:// creativecommons.org/licenses/by-nc/4.0/

\section{REFERENCES}

1. Bonadonna P, Schiappoli M, Dama A, et al. Is hymenoptera venom allergy an occupational disease? Occup Environ Med 2008:65:217-18.

2. Gregorian $\mathrm{Ch}$, Galatas ID, Klamouris $\mathrm{Ch}$, et al. Insect venom allergy in Greek adults. Allergy 1997;52:51-7.

3. Müller UR. Insect sting allergy. Stuttgart, Germany: Gustav Fischer, 1990.

4. Incorvaia $\mathrm{C}$, Mauro M, Pravettoni $\mathrm{V}$, et al. Hypersensitivity to hymenoptera venom: advances in diagnosis and implications for treatment. Recent Pat Inflamm Allergy Drug Discov 2011;5:128-35.

5. Bonifazi $F$, Jutel M, Bilò MB, et al. Prevention and treatment of hymenoptera venom allergy. Allergy 2005:60:1459-70.

6. Kahan E, Ben-Moshe R, Derazne E, et al. The impact of Hymenoptera venom allergy on occupational activities. Occup Med 1997;47:273-6.

7. Bilo BM, Rueff $\mathrm{F}$, Mosbech $\mathrm{H}$, et al. Diagnosis of Hymenoptera venom allergy. Allergy 2005;60:1339-49.

8. Eisner MD, Yelin EH, Katz PP, et al. Risk factors for work disability in severe adult asthma. Am J Med 2006;119:884-91.

9. Torén K, Zock JP, Kogevinas M, et al. An international prospective general population-based study of respiratory work disability. Thorax 2009;64:339-44.

10. Lemeshow S, Hosmer DW Jr. A review of goodness of fit statistics for use in the development of logistic regression models. Am J Epidemiol 1982;115:92-106.

11. De la HozCaballer B, Rodriguez M, Fraj J, et al. Allergic rhinitis and its impact on work productivity in primary care practice and a comparison with other common diseases: the cross-sectional study to evaluate work productivity in allergic rhinitis compared with other common diseases (CAPRI) study. Am J Rhinol Allergy 2012;26:390-4.

12. Virchow JC, Kay S, Demoly P, et al. Impact of ocular symptoms on quality of life $(\mathrm{QoL})$, work productivity and resource utilisation in allergic rhinitis patients-an observational, cross sectional study in four countries in Europe. J Med Econ 2011;14:305-14.

13. Murgia N, Torén $\mathrm{K}$, Kim JL, et al. Risk factors for respiratory work disability in a cohort of pulp mill workers exposed to irritant gases. BMC Public Health 2011;11:689.

14. Solomon C, Poole J, Palmer KT, et al. Health-related job loss: findings from a community-based survey. Occup Environ Med 2007;64:144-9.

15. Ozdemir C, Kucuksezer UC, Akdis M, et al. Mechanism of immunotherapy to wasp and bee venom. Clin Exp Allergy 2011;41:1226-34

16. Bilò MB. Anaphylaxis caused by hymenoptera stings: from epidemiology to treatment. Allergy 2011;66:35-7.

17. Krishna MT, Ewan PW, Diwakar L, et al. Diagnosis and management of hymenoptera venom allergy: British Society for Allergy and Clinical Immunology (BSACl) guidelines. Clin Exp Allergy 2011;41:1201-20.

18. Van Oostrom SH, Driessen MT, de Vet HC, et al. Workplace interventions for preventing work disability. Cochrane Database Syst Rev 2009;(2):CD006955.

19. Blanc PD, Ellbjar S, Janson C, et al. Asthma-related work disability in Sweden. The impact of workplace exposures. Am J Respir Crit Care Med 1999;160:2028-33.

20. Oude Elberink JN, de Monchy JG, Golden DB, et al. Development and validation of a health-related quality-of-life questionnaire in patients with yellow jacket alergy. J Allergy Clin Immunol 2002;109:162-70.

21. Omachi TA, Claman DM, Blanc PD, et al. Obstructive sleep apnea: a risk factor for work disability. Sleep 2009;32:791-8.

22. Teschke K, Smith JC, Olshan AF. Evidence of recall bias in volunteered vs. prompted responses about occupational exposures. Am J Ind Med 2000;38:385-8. 